\title{
2 Andrologie in der Praxis
}

\author{
Hermann J. Berberich
}

In den letzten Jahrzehnten hat sich das Tätigkeitsgebiet des niedergelassenen Urologen erheblich verändert. Ursprünglich ein Kind der Chirurgie, hat es die Urologie verstanden, sich neue Fachgebiete anzueignen. Hierzu gehört insbesondere die Andrologie. Diese hat mittlerweile einen festen Platz in der Urologie gefunden. Seit der Aufnahme der Zusatzqualifikation Andrologie in die ärztliche Weiterbildungsordnung 2003 konnten 1.100 Fachärzte für Urologie die Zusatzbezeichnung „Andrologie“ erwerben und somit eine flächendeckende andrologische Versorgung sicherstellen. Die überwiegende Mehrheit der Patienten mit andrologischen Problemen wendet sich heute an eine urologische Praxis. Die Erfahrung zeigt, dass immer mehr Männer, insbesondere mit sexuellen Funktionsstörungen, gezielt einen Urologen aufsuchen, der eine andrologische Zusatzqualifikation besitzt.

\subsection{Sexuelle Funktionsstörungen}

Angesichts der Tatsache, dass 52\% der Männer zwischen 40 und 70 Jahren zumindest an leichtgradigen Erektionsstörungen leiden (Feldmann et al. 1994), wundert es nicht, dass inzwischen die erektile Dysfunktion das häufigste andrologische Krankheitsbild ist, weshalb Männer eine urologische Praxis aufsuchen. Seit der Einführung von Viagra vor mehr als 10 Jahren und der damit verbundenen Enttabuisierung trauen sich immer mehr Männer, häufig gedrängt von ihren Partnerinnen, aus diesem Grund ärztlichen Rat nachzusuchen. Der deutliche Anstieg der Erektionsstörungen mit dem Alter legt nahe, dass organische Krankheiten, die ebenfalls mit dem Alter zunehmen, eine wichtige Rolle spielen. Laut der Kölner Studie (Braun et al. 200o) besteht eine hohe Komorbidität von Erektionsstörungen mit dem Bluthochdruck, Diabetes mellitus, operativen Eingriffen im kleinen Becken sowie den sog. Lower urinary tract symptoms (LUTS). Hierbei handelt es sich um Blasenentleerungsstörungen, die im Wesentlichen auf eine altersbedingte Prostatahyperplasie zurückzuführen sind. Vor allem bei Männern in der Altersgruppe zwischen 50 und 59 Jahren können Erektionsstörungen die Vorboten einer koronaren Herzerkrankung sein (Speed et al. 2003). 
Deshalb sollten ältere Männer mit Erektionsstörungen immer auch kardiologisch abgeklärt werden. Die Gefahr, beim Geschlechtsverkehr einen Herzinfarkt zu erleiden, ist allerdings gering (Muller et al. 1996). Nicht selten verbirgt sich jedoch hinter einer angeblichen Erektionsstörung in Wirklichkeit eine larvierte Appetenzstörung, was sich zum Teil erst nach erfolgloser Anwendung eines PDE-5-Hemmers offenbart. Mitunter wird dann vom Patienten beklagt, dass das eingenommene Medikament nicht wirke oder er vermutet eine schwere organische Ursache seiner Erektionsprobleme. Denn einfach „keine Lust zu haben“ ist noch weniger mit dem sexuellen Selbstkonzept vieler Männer vereinbar als das Vorliegen einer genitalen Funktionsstörung. Appetenz- und Erektionsstörungen treten gerade bei älteren Männern gehäuft gemeinsam auf. Dabei ist zu klären, ob der Appetenzmangel situativ oder generalisiert auftritt und über welchen Zeitraum der Patient die Störung beklagt. Nicht selten ist die Appetenzstörung Zeichen einer Depression oder Folge eines Erschöpfungszustandes. Organische Ursachen wie Testosteronmangel, Hyperprolaktinämie oder Medikamente sind zwar differenzialdiagnostisch bedeutsam, werden jedoch häufig in ihrer Bedeutung überschätzt.

Bei jüngeren Männern unter 45 Jahren sind sexuelle Appetenzstörungen selten das Hauptproblem, sondern eher eine Reaktion auf Erektions- oder Orgasmusstörungen. Ist dies dennoch der Fall, so ist darauf zu achten, ob deviante Sexualpräferenzen, massive Sexualängste oder sexuelle Orientierungskonflikte die Ursache sind (Beier et al. 2005).

Der vorzeitige Orgasmus (Ejaculatio praecox) ist die häufigste Sexualstörung des Mannes. Seine Prävalenz liegt in allen Altersgruppen zwischen 20 und 25\%. Auf Dauer ist die Ejaculatio praecox für die Partnerschaft die schädlichste sexuelle Funktionsstörung. Nicht selten gibt die Partnerin „ihm“ wegen „mangelnder Selbstbeherrschung“ die Schuld für das unbefriedigende Sexualleben.

Die Definition, wann man von einem frühem Orgasmus sprechen kann, ist nach wie vor kontrovers. Seine Bewertung unterliegt sowohl intraindividuellen als auch kulturellen Schwankungen. Grundsätzlich wird zwischen einem „primären“ und einem „sekundären“ vorzeitigen Orgasmus unterschieden. Beide Formen können zusammen mit einer Erektions- oder Appetenzstörung einhergehen. Einige Autoren (Waldinger 2002) vertreten die These, dass es sich beim lebenslangen „primären vorzeitigen Orgasmus“ um eine angeborene Störung der serotonergen Leitungsbahnen, die die Ejakulation steuern, handelt. Da bei keiner Primatenart ein lang andauernder Geschlechtsverkehr bekannt ist, kann angenommen werden, dass eine schnelle Ejakulation ursprünglich das biologisch „Normale“ ist.

Bekanntlich unterliegt menschliches Sexualverhalten einem kulturellen Transformationsprozess. Zumindest in unserem Kulturkreis versuchen Männer, die Ejakulation hinauszuzögern. Aus unterschiedlichen Gründen sind viele hierzu nicht oder vorübergehend nicht in der Lage

$\mathrm{Zu}$ einer möglichen biologischen Disposition kommen beim konkreten Verlauf, ein angstbetonter sympathikobetonter Shift sowie die partnerschaftliche Belastung hinzu. Es entwickelt sich nicht selten ein „Teufelskreis“ aus dem Erleben eines vorzeitigen Samenergusses, der Angst vor sexueller Erregung, dem Einsatz kognitiver Ablenkungsmanöver, einer erhöhten Anspannung und einer schnelleren Auslösung des Orgasmusreflexes. 
Der ausbleibende bzw. gehemmte Orgasmus ist zwar seltener als der vorzeitige Orgasmus, aber mit einer Prävalenz von 8\% häufiger als gemeinhin angenommen (Laumann et al. 1999). Nach eigenen klinischen Erfahrungen haben vor allem zwanghaft veranlagte Männer Probleme zu einem Orgasmus zu kommen. Sie haben Angst vor einem Kontrollverlust, konzentrieren sich in erster Linie beim Geschlechtsverkehr darauf, es der Partnerin „Recht zu machen“ und setzen sich selbst unter Leistungsdruck. Da sie einfach nicht loslassen können, leiden sie häufig auch an funktionellen Blasenentleerungsstörungen ohne organpathologischen Befund. Wie bei der erektilen Dysfunktion kann sich hinter einem ausbleibenden Orgasmus in Wirklichkeit auch eine Appetenzstörung verbergen. Dies wird man nur durch eine sorgfältige Sexualanamnese, d.h. durch eine genaue Analyse des „sexuellen Drehbuchs“ herausfinden können.

Weitere Störungsbilder sind der schmerzhafte Orgasmus infolge einer Beckenbodenmyalgie und dem daraus resultierenden chronischen Beckenschmerz (CPPS) oder die retrograde Ejakulation, z.B. nach einer Prostataoperation. Beide können das sexuelle Erleben des Mannes negativ beeinflussen.

Tritt allerdings eine Orgasmushemmung nach langjähriger ungestörter Sexualität auf, müssen neurologische Störungen wie der M. Parkinson oder die Multiple Sklerose in Betracht gezogen werden.

Ebenso kann ein langjähriger Alkohol- oder Drogenmissbrauch die Ursachen darstellen.

\section{Diagnostik und Therapie sexueller Funktionsstörungen}

Kernstück der sexualmedizinischen Diagnostik ist eine ausführliche Sexualanamnese (s. Tab. 1), die sowohl die biologischen, die psychischen als auch die sozialen Faktoren und deren individuelle Gewichtung und Vernetzung bei den sexuellen Störungen im Einzelfall erfassen sollte. Hierzu gehört selbstverständlich auch die Erfassung der allgemeinen Krankengeschichte sowie der aktuellen Medikamenteneinnahme.

\section{Tab. 1 Anforderungen und Besonderheiten der Sexualanamnese}

\footnotetext{
Überwinden der Sprachlosigkeit (Sexualität als Tabuthema)

Wahrnehmung des Leidensdrucks

bisherige Lösungsversuche

Auswirkung der Sexualstörung auf die Beziehung (Reaktion der Partner/in) bisherige Erkrankungen, Medikamente

Bedeutung der drei Dimensionen (Reproduktion, Lust und Beziehung)

Erfüllung des psychosozialen Grundbedürfnisses nach Nähe, Akzeptanz und Geborgenheit im Rahmen der Sexualität,

Mikroanamnese des sexuellen Verhaltens und Erlebens (genitale-, nicht-genitale Sexualität, Selbstbefriedigung, Präferenzstruktur [Geschlecht, Alter, Praktiken], Fantasie, Verhalten, Selbstkonzept)

Lebensgeschichte und soziosexuelle Entwicklung
} 
Liegt der Verdacht auf eine organische (Mit-)Ursache einer sexuellen Funktionsstörung vor, muss dieser auf jeden Fall abgeklärt werden (s. Tab. 2).

Da sich Sexualität in Beziehungen widerspiegelt und Störungen immer Auswirkungen auf die Beziehung haben, ist eine frühzeitige Einbindung der Partnerin, des Partners sowohl in den diagnostischen Prozess als auch in die Therapie anzustreben. Dies ist auch beim Einsatz von Medikamenten (s. Tab. 3) anzustreben. Leider geschieht dies im urologischen Alltag nur allzu selten. Der ausschließliche Blick auf die „gestörte Organfunktion“ ist unzureichend, da wesentliche Aspekte der gestörten Sexualität (z.B. Verursachungsmechanismen, Auswirkungen etc.) nicht erfasst werden würden.

\section{Tab. 2 Untersuchungen bei Verdacht auf organische Ursachen einer Erektionsstörung}

\begin{tabular}{l|l}
$\begin{array}{l}\text { Basisdiagnostik } \\
\text { Stufe I }\end{array}$ & $\begin{array}{l}\text { körperliche Untersuchung, Blutdruck, Puls, ggf. EKG } \\
\text { Laboruntersuchungen } \\
\text { Elektrolyte, kleines Blutbild, Blutfette, Nüchtern-Glukose, Nieren- und } \\
\text { Leberwerte, Testosteron. }\end{array}$ \\
\hline $\begin{array}{l}\text { nicht-invasive andrologische } \\
\text { Diagnostik }\end{array}$ & $\begin{array}{l}\text { Pharmakodoppler- und Duplexsonografie zur Beurteilung der funktionellen } \\
\text { Kapazität der Penisarterien, aufsteigende SKAT-Testung zur Beurteilung der } \\
\text { Stufe II }\end{array}$ \\
$\begin{array}{l}\text { kavernösen Funktionsfähigkeit, ggf. Schwellkörper-EMG } \\
\text { invasive andrologische } \\
\text { Diagnostik }\end{array}$ & $\begin{array}{l}\text { Röntgendarstellung der Penisgefäße. Nur erforderlich, wenn definitiv ein } \\
\text { operativ-rekonstruktives Operationsverfahren, z.B. eine Revaskularisie- }\end{array}$ \\
\hline Stufe III & rungsoperation geplant ist.
\end{tabular}

Tab. 3 Medikamente als möglicher Bestandteil einer sexualmedizinischen Behandlung

\begin{tabular}{|c|c|c|c|}
\hline Substanz & Applikation & Wirkmechanismus & Indikation \\
\hline $\begin{array}{l}\text { Sildenafil } \\
\text { Vardenafil } \\
\text { Tadalafil }\end{array}$ & oral & $\begin{array}{l}\text { selektiver PDE-5 Hemmer, relaxiert } \\
\text { die Schwellkörpermuskulatur durch } \\
\text { Hemmung des cGMP-Abbaus }\end{array}$ & Erektionsstörungen \\
\hline Prostaglandin E1 & $\begin{array}{l}\text { intracavernös (SKAT) } \\
\text { transurethral } \\
\text { (MUSE) }\end{array}$ & $\begin{array}{l}\text { Gewebshormon, relaxiert die } \\
\text { Schwellkörper-muskulatur }\end{array}$ & Erektionsstörungen \\
\hline Dapoxetin & oral & $\begin{array}{l}\text { Serotoninwiederaufnahmehemmer, } \\
\text { Stimulation der zentralen } \\
\text { Serotoninrezeptoren }\end{array}$ & vorzeitige Ejakulation \\
\hline Testosteron & $\begin{array}{l}\text { oral } \\
\text { transkutan } \\
\text { intramuskulär }\end{array}$ & $\begin{array}{l}\text { zentral, Freisetzung und Speicherung } \\
\text { proerektiler Neurotransmitter } \\
\text { (Oxytocin, Dopamin, N0), } \\
\text { Testosteronmangel führt zur } \\
\text { Apoptose der glatten Schwellkörper- } \\
\text { muskelzelle }\end{array}$ & $\begin{array}{l}\text { nachgewiesener } \\
\text { Hypogonadismus mit } \\
\text { Auswirkung auf } \\
\text { Appetenz und Erektion }\end{array}$ \\
\hline
\end{tabular}




\subsection{Entzündliche Erkrankungen des äußeren Genitale und der Prostata}

Eine Harnröhrenentzündung (Urethritis) kann sowohl durch spezifische (Gonokokken) als auch durch unspezifische Erreger (Chlamydien, Mykoplasmen, Trichomonaden, Candida, Staphylokokken, Enterobakterien) verursacht werden. Die Patienten klagen über weißlichen bis grünlichen Ausfluss (vor allem am Morgen), Brennen in der Harnröhre und eine gehäufte, schmerzhafte Miktion. Diagnostisch ist eine 3- bzw. 4-Gläserprobe und der Harnröhrenabstrich mit Anlage einer Kultur erforderlich. In der Regel wird man eine sogenannte kalkulierte antibiotische Therapie z.B. mit einem Gyrasehemmer oder einem Tetracyclin einleiten, die mithilfe eines Antibiogramms ggf. verändert wird. Je nach Erreger sollte eine Partnerbehandlung veranlasst werden. Eine unbehandelte Urethritis ist nicht selten Verursacher einer Nebenhodenentzündung (Epididymitis). Anzeichen hierfür ist ein schmerzhaft geschwollenes und gerötetes Skrotum. Mitunter strahlen die Schmerzen in die Leiste aus. Ebenso können Fieber und Schmerzen beim Urin lassen vorkommen. Differenzialdiagnostisch ist vor allem bei jungen Männern eine Hodentorsion auszuschließen, die innerhalb eines kurzen Zeitfensters behoben werden muss, um eine Hodenschädigung zu vermeiden.

Ferner sollte sonografisch der Ausschluss eines Nebenhodenabszesses, eine sog. Fokussuche (Sonografie der Nieren, der Prostata) und Labor/Urinanalyse erfolgen.

Die Orchitis, d.h. die Entzündung des gesamten Hodens, tritt zumeist infolge einer allgemeinen Infektion auf. Ihre Symptome sind: schmerzhaft geschwollene Skrotalhälfte, Rötung, Fieber, allgemeines Krankheitsgefühl. Neben der körperlichen Untersuchung sollte unbedingt eine Ultraschalluntersuchung des Hodens zum Ausschluss eines Hodentumors oder eines Hodenabszesses erfolgen. Differenzialdiagnostisch ist darüber hinaus eine Hodentorsion, eine Parotitis epidemica, eine Uro-TBC und ggf. eine Syphilis abzuklären.

Schmerzlose Veränderungen der Skrotalhaut werden häufig durch Pilze verursacht. Die Patienten klagen über schuppige Hautveränderungen und Juckreiz. Die diagnostische Abklärung erfolgt durch spezielle Nährmedien, zur Behandlung eignen sich antimykotische Salben.

Die Urogenital-TBC ist mit 30-40\% die häufigste extrapulmonale Organmanifestation, die sich erst nach vielen Jahren aus einer Lungen-TBC entwickelt. Urlaubsreisen in ferne Länder, Migration und das neue offene Europa haben zu einem erneuten Anstieg der TBC auch in Deutschland geführt. Wegen der uncharakteristischen Symptomatik wird die Urogenital-TBC auch heute noch häufig sehr spät entdeckt. Eine bereits früher durchgemachte ТВC und Symptome wie subfebrile Temperaturen, Nachtschweiß, eine antibiotikaresistente Zystitis ohne Keimnachweis (sterile Leukozyturie), persistierende Mikro- oder Makrohämaturie, derbe indolente Veränderungen an Samenstrang, Nebenhoden und Prostata sollten zur entsprechenden Diagnostik veranlassen. Diese besteht vor allem in einer Untersuchung des Urins und ggf. des Ejakulats auf säurefeste Stäbchen und die sonografische Untersuchung der Harnwege zum Nachweis charakteristischer Veränderungen (Cavernenbildung, Hydronephrosen, Schrumpfniere). Therapeutischer Standard ist heute eine Kombinationsbehandlung mit vier Tuberkulostatika, die bei guter Patientencompliance auch ambulant durchgeführt werden kann. 
Unter dem Begriff Prostatasyndrom wird eine ganze Reihe von urogenitalen, perinealen und perianalen Beschwerden subsumiert. Diese reichen von starken, akuten Schmerzen bis zu mäßig-diffusen Beschwerden, die nur schwer zuzuordnen sind und nicht selten fälschlicherweise der Prostata zugeschrieben werden. Die eher seltene akute Prostatitis ist leicht zu diagnostizieren. Sie ist gekennzeichnet durch eine starke schmerzhafte Schwellung der Prostata, Fieber, eine Leukozyturie und Bakterurie. Liegt ein Abszess vor, findet man palpatorisch eine Fluktuation und im transrektalen Ultraschall hypo- bzw. areflexive Zonen mit Wandverdickung oder perifokalen Ödemen. Kommt es durch die Prostataschwellung zu einer Harnverhaltung, ist eine suprapubische Urinableitung erforderlich. Auf keinen Fall sollte der Patient transurethral katheterisiert werden. Bei einer akuten Prostatitis ist eine hochdosierte Antibiotikatherapie unbedingt erforderlich. Bei der chronischen bakteriellen Prostatitis findet sich häufig eine akute Prostatitis, eine Epididymitis oder eine Urethritis in der Vorgeschichte. Beweisend sind entsprechende Entzündungsparameter im Exprimaturin im Rahmen einer 2- oder 4-Cläserprobe. Als weiteres Diagnosekriterium gilt heute auch eine erhöhte Leukozytenzahl im Ejakulat.

Ob die von den Patienten häufig geschilderten eher diffusen Beschwerden wie Druckgefühl im Damm, Beschwerden in den Leisten mit Ausstrahlung in den Hoden, vermehrter Harndrang, erschwerte Blasenentleerung, Brennen in der distalen Harnröhre, Nachträufeln, Druckgefühl oder Brennen hinter dem Schambein, Spannungsgefühl in der Dorsolumbal- und Sakralregion in direktem Zusammenhang mit entzündlichen Veränderungen in der Prostata stehen, wird insbesondere von psychosomatischer Seite bezweifelt, da andere entzündliche Erkrankungen der Prostata wie die granulomatöse Prostatitis meistens ohne Beschwerden einhergehen und häufig nur im Rahmen einer Prostatabiopsie zum Ausschluss eines Prostatakarzinoms diagnostiziert werden (Berberich et al. 2004). Vielmehr scheint es sich bei den oben genannten Beschwerden eher um die Folgen einer erhöhten Körperaufmerksamkeit gegenüber dieser Körperregion nach einer früher abgelaufenen Entzündung zu handeln. Aufgrund einer chronischen Anspannung der Beckenbodenmuskulatur kommt es schließlich zur Ausbildung einer schmerzhaften Beckenbodenmyalgie und zur Entwicklung eines chronischen Beckenschmerzsyndroms (CPPS). Einen Patienten allein aufgrund der genannten Beschwerden ohne Nachweis von Entzündungsparametern antibiotisch zu behandeln, ist nicht zuletzt aufgrund der rapide zunehmenden Resistenzbildung gegen Antibiotika abzulehnen. Vielmehr ist es notwendig, dem Patienten die psycho-physiologischen Zusammenhänge zu erklären, ihm die nicht seltene Angst vor einem Prostatakarzinom zu nehmen und ihn zu Eigenaktivitäten wie z.B. der Erlernung eines Entspannungsverfahrens zu bewegen. Besteht jedoch der Hinweis, dass sich hinter den Symptomen eine neurotische Erkrankung, z.B. eine lavierte Depression, verbirgt, sollte der Patient dazu motiviert werden, sich in psychotherapeutische Behandlung zu begeben. Deshalb ist es verwirrend, dass auch die neue Prostatitisklassifikation (s. Tab. 4) den chronischen Beckenschmerz nach wie vor mit aufführt. 
Tab. 4 NIH - Prostatitis-Klassifikation

\begin{tabular}{|c|c|c|}
\hline Kategorie & Bezeichnung & Erläuterung \\
\hline I & akute bakterielle Prostatitis & akute bakterielle Infektion \\
\hline ॥ & chronische bakterielle Prostatitis & chronische bakterielle Infektion \\
\hline III & chronische abakterielle Prostatitis/CPPS & keine nachweisbaren Erreger \\
\hline IIla & $\begin{array}{l}\text { entzündliches chronisches Schmerzsyndrom } \\
\text { des Beckens }\end{array}$ & $\begin{array}{l}\text { erhöhte Leukozytenzahl im Prostataexprimat, } \\
\text { Exprimaturin und Ejakulat }\end{array}$ \\
\hline IIlb & $\begin{array}{l}\text { nicht-entzündliches chronisches Schmerz- } \\
\text { syndrom des Beckens }\end{array}$ & $\begin{array}{l}\text { keine erhöhte Leukozytenzahl im Prostata- } \\
\text { exprimat Exprimaturin und/oder Ejakulat }\end{array}$ \\
\hline \multirow[t]{2}{*}{ IV } & \multirow[t]{2}{*}{ asymptomatische entzündliche Prostatitis } & $\begin{array}{l}\text { keine Symptome, Nachweis von Entzündungs- } \\
\text { zellen in der Prostatabiopsie }\end{array}$ \\
\hline & & $\begin{array}{l}\text { erhöhte Leukozytenzahl im Prostataexprimat, } \\
\text { Exprimat und/oder Ejakulat }\end{array}$ \\
\hline
\end{tabular}

\subsection{Sexuell übertragbare Krankheiten}

Trotz intensiver Aufklärungsbemühungen, insbesondere im Zusammenhang mit der HIV-Erkrankung, durch Institutionen wie zum Beispiel die Bundeszentrale für gesundheitliche Aufklärung (BZCA) scheinen die sexuell übertragbaren Erkrankungen weiterhin zuzunehmen. Laut Schätzung der WHO gibt es weltweit jährlich 340 Millionen Krankheitsfälle. Diese Zahlen beziehen sich ausschließlich auf heilbare STDInfektionen wie Syphilis, Gonorrhoe, Chlamydien- und Trichomonadeninfektion. In allen europäischen Ländern nehmen insbesondere die „klassischen Geschlechtskrankheiten“ wieder zu. Allein in Deutschland verdoppelten sich die Syphilis-Erkrankungen zwischen den Jahren 2000 und 2002.

Kondylome (genitale Feigwarzen) sind bei Männern die häufigste sexuell übertragene Erkrankung. Vom Verursacher, dem humanen Papillomavirus (HPV) konnten inzwischen mehr als 100 Typen identifiziert werden. Die Diagnose ist einfach. Beim Bestreichen der verdächtigen Partien mit 5\%-Essigsäure färben sich Kondylome weiß. Ihre Behandlung ist sowohl konservativ (Podopyllotoxin, Imiquimod) als auch chirurgisch (Elektro- oder Laserkoagulation) möglich. Ausgedehnte schwer behandelbare Kondylomerkrankungen finden sich aufgrund einer T-Zell-Funktionsstörung bei HIV-infizierten Patienten. Es ist ratsam, bei Patienten mit Kondylomen sowohl eine LUES-Serologie als auch einen HIV-Test vorzunehmen.

Die zweithäufigste STD-Erkrankung ist die Infektion durch das intrazelluläre Bakterium Chlamydia Trachomatis. Beim Mann verursacht es vor allem Entzündungen der Harnröhre und des Nebenhodens. Da bei einer Infektion Chlamydien sich an die Spermatozoen anheften können, können sie bei ungeschütztem Verkehr bei der Frau eine Eileiterentzündung und einen Tubenverschluss mit konsekutiver Sterilität hervorrufen.

Zur diagnostischen Methode der Wahl hat sich der DNS-Nachweis auf Basis des PCR-Verfahrens entwickelt. Hierzu ist ein spezieller Harnröhrenabstrich beim Mann erforderlich. Die Bestimmung aus dem Erststrahl-Urin ist weniger zuverlässig. 
Die Behandlung ist relativ einfach. Die deutsche STD-Gesellschaft empfiehlt die Gabe von $200 \mathrm{mg}$ Doxycyclin über 7 Tage oder eine Einmalgabe von Azithromycin 1.00o mg, alternativ ist auch eine Behandlung mit Tetracyclin-HCL, Erythromycin oder einem Gyrasehemmer (Ciprofloxacin, Levofloxacin) möglich.

Die Infektion mit Neisseria gonorrhoeae ist zwar in Europa rückläufig, sie gehört aber nach wie vor zu den häufigsten Infektionskrankheiten weltweit. Insbesondere nach Urlaubsaufenthalten (Asien, Fernost) findet sich mancher Patient mit typischen Gonorrhoe-Beschwerden (Entzündung der vorderen Harnröhre, grünlicher Ausfluss) in der urologischen Praxis ein.

Ferner kann es zu einer Prostata- oder Nebenhodenentzündung kommen. Der Nachweis von Neisseria gonorrhoeae erfolgt im Ausstrich sowie in der Kultur.

Die Behandlung mit Penicillin gilt wegen der vielen resistenten Keime als obsolet, stattdessen empfiehlt die Deutsche STD-Gesellschaft die Einmalgabe von Ceftriaxon $250 \mathrm{mg}$ i.m. Alternativ ist die orale Verabreichung von Cefixim oder Azithromycin möglich.

Das Herpes-simplex Virus wird ebenfalls durch Sexualkontakte übertragen und kann akute und rezidivierende genitale Erkrankungen bewirken. Beim Mann findet man 2-3 mm große Bläschen auf der Glans penis und/oder am inneren Vorhautblatt.

Therapie der Wahl ist die lokale Behandlung mit Aciclicovir-Creme oder die systemische Gabe von Aciclicovir, Famciclovir bzw. Valaciclovir. Bei starken Schmerzen kann als Begleitmedikation ein Antiphlogistikum gegeben werden.

Seit der Öffnung des „Eisernen Vorhangs“ ist wieder eine Zunahme der Syphilis-Infektionen zu verzeichnen. Die überwiegende Zahl der Neuinfektionen ist auf Kontakte in Osteuropa oder mit von dort stammenden Personen zurückzuführen. Die Übertragung erfolgt nur bei direktem körperlichem Kontakt.

Die Syphilis verläuft in drei Stadien.

- Im Stadium I entwickelt sich an der Infektionsstelle in der Regel im Genitalbereich, nach ungefähr. drei Wochen ein sogenannter Primäraffekt.

- Im Stadium II kommt es durch hämatogene Ausbreitung des Erregers im gesamten Körper zu einer generellen Infektionskrankheit, es bildet sich z.B. ein makulös fleckiges Exanthem am Stamm und in den Flanken.

- Im Stadium III können alle Organsysteme betroffen sein (tastbare Lymphknotenschwellung, Leberschwellung, hirnorganische Veränderungen).

In der Regel werden Patienten im Stadium I die urologische Praxis aufsuchen, im Stadium II eher selten. Die Behandlung ist relativ unproblematisch. Bei einer Frühsyphilis ist die einmalige Gabe eines Depot-Penicillinpräparates i.m. ausreichend. Penicillin-resistente Stämme von Treponoma Pallidum sind bislang nicht bekannt.

\section{HIV und Hepatitis C zählen zu den "STD-Krankheiten im weiteren Sinne“.}

Mitunter kommt es in der urologischen Praxis vor, dass eine HIV-Infektion zufällig im Rahmen der präoperativen Labordiagnostik vor einer urologischen Operation entdeckt wird. 


\subsection{Unerfüllter Kinderwunsch}

Von unerfülltem Kinderwunsch kann man sprechen, wenn es bei ungeschütztem regelmäßigem Geschlechtsverkehr (3- bis 4-mal pro Woche) innerhalb eines Jahr zu keiner Konzeption gekommen ist. Die Prävalenz liegt bei ca. 14\%. Die Zahl der auf Dauer ungewollt kinderlosen Paare liegt mit 3\% deutlich niedriger (Brähler u. StöbelRichter 2002).

Der Kinderwunsch ist stark von emotionalen Aspekten geprägt. Bei Männern ist er nicht selten mit Problemen der Selbstwertregulation verbunden, dies gilt vor allem für Männer mit schlechter Fertilitätsprognose. Diese Selbstwertproblematik führt dazu, dass viele Männer sich erst nach erheblichem Drängen ihrer Partnerinnen andrologisch untersuchen lassen. Sie überlassen es ihren Frauen, den Untersuchungstermin zu vereinbaren, mitunter versäumen sie den vereinbarten Termin oder die Befundbesprechung. Bei nahezu der Hälfte der Paare ist die Kinderlosigkeit androgen zumindest mitbedingt.

$\mathrm{Zu}$ einer andrologischen Basisdiagnostik gehören eine sorgfältige Krankheits- und Sexualanamnese einschließlich der Erfassung der aktuellen Medikamenteneinnahme (zahlreiche Medikamente können sich auf die Spermatogenese auswirken). Ferner ist eine vollständige körperliche Untersuchung (einschl. der Untersuchung der männlichen Brust, der Körper- und Schambehaarung, des äußeren Genitale und der Prostata) erforderlich. Hierfür muss sich der Patient vollständig ausziehen. Zur Routinediagnostik gehört außerdem eine Ejakulatanalyse nach WHO-Kriterien.

Eine sonografische Untersuchung der Hoden sowie eine genitale Dopplersonografie zum Ausschluss einer Varicozele sind ratsam. Fast alle urologischen Praxen sind entsprechend apparativ ausgestattet.

Weiterführende Untersuchungen wie z.B. eine Hormonanalyse oder die Suche nach Spermatozoenantikörper sind erst erforderlich, wenn im Rahmen der Basisdiagnostik ein pathologischer Befund erhoben wurde. In der Altersgruppe zwischen 20 und 50 Jahren gibt es keinen Hinweis auf eine altersbedingte Fertilitätseinschränkung, während ca. 30\% der Männer über 6o Jahre und 50\% der Männer über 80 Jahre infertil sind. Sind entzündliche Erkrankungen der Genitalorgane für eine Spermatogenesestörung verantwortlich, was bei 9-15\% der Patienten der Fall ist, sind diese entsprechend zu behandeln. Bei weniger als 10\% sind hormonelle Störungen die Ursachen. Beim sogenannten sekundären Hypogonadismus aufgrund eines hypothalamischen GnRH-Mangel und einem hypophysären LH- und FSH-Mangel ist eine Kombinationsbehandlung mit HCG plus HMC (bzw. FSH) oder eine zyklische GnRH-Applikation (Minipumpe) zur Verbesserung der Ejakulatqualität möglich.

\subsection{Familienplanung}

Nach wie vor ist kein Medikament zur Empfängnisverhütung für den Mann auf dem Markt. Übernimmt der Mann die Verantwortung für die Reproduktionskontrolle, bleibt ihm nur die mechanische Verhütung durch ein Kondom oder bei abgeschlossener Familienplanung die Vasektomie als Mittel der Wahl. Während 1992 nur o,5\% der Männer im zeugungsfähigen Alter sterilisiert waren, stieg die Zahl bis 2006 auf 2\% (zum Vergleich: bei Frauen sind es 8\%). Der Eingriff ist relativ einfach und kann 
ambulant in Lokalanästhesie durchgeführt werden. Der sogenannte Pearlindex liegt bei o,25\%, das heißt einer von vierhundert Männern bleibt trotz Sterilisation zeugungsfähig.

Es gibt mehrere operative Techniken der Vasektomie. Die bekannteste minimal invasive Methode ist die aus China stammende „No scalpel vasectomy“. Weitere Methoden sind die Fulgurationstechnik, die Ligaturtechnik (konventionelle Vasektomie), die open ended vasectomy und die Cliptechnik. Für alle Methoden gilt, dass auch nach mehreren Wochen und Monaten befruchtungsfähige Spermatozoen im Ejakulat vorhanden sein können. Deshalb ist es erforderlich, eine ganze Zeit lang zusätzliche Verhütungsmethoden anzuwenden. Erst wenn in zwei im Abstand von ca. 4 Wochen durchgeführten postoperativen Ejakulatuntersuchungen keine Spermien mehr zu finden sind, kann auf weitere Verhütungsmaßnahmen verzichtet werden. $\mathrm{Zu}$ den möglichen Komplikationen nach Vasektomie gehören Hämatome, Nebenhodenentzündungen, skrotale Abszesse, Infektionen, Spermgranulome, Verwachsungen zwischen Samenleiter und Haut. Erfreulicherweise sind die Komplikationsraten seit Jahren rückläufig (Gingell et al. 2001)

\subsection{Von der Reparatur zur Vorsorgemedizin: Die urologisch-andrologische Früherkennung}

Nach wie vor gelten deutsche Männer als Vorsorgemuffel. Während ca. 50\% aller Frauen regelmäßig die gynäkologische Krebsfrüherkennungsuntersuchungen, tun dies nur 15\% der Männer. Obwohl mit fast 50.000 Neuerkrankungen pro Jahr das Prostatakarzinom fast genauso häufig ist wie der weibliche Brustkrebs mit ca. 55.000 (Arbeitsgemeinschaft Bevölkerungsbezogener Krebsregister in Deutschland 2004).

Nach wie vor sterben Männer durchschnittlich sechs Jahre früher als Frauen. Wie die bayrische Klosterstudie beweist, hat dies keine biologische Ursache, sondern ist hauptsächlich auf das männliche Risikoverhalten zurückzuführen.

Während Frauen ab dem 2o. Lebensjahr, bezahlt von der Krankenkasse, regelmäßig zur gynäkologischen Früherkennungsuntersuchung gehen können, gibt es für den Mann zwischen 18 und 35 Jahren keine Vorsorgeuntersuchung, sieht man einmal von der Musterung ab. Mit der Aussetzung der Wehrpflicht soll auch diese Untersuchung wegfallen. Dabei werden gerade in dieser Altersgruppe die Weichen für die Gesundheit ab dem 5o. Lebensjahr gestellt und die häufigsten Hodentumoren diagnostiziert. Mit 4.35o Neuerkrankungen im Jahr ist dieser fast genauso häufig wie die Leukämie mit jährlichen 5.50o Neuerkrankungen. Im Gegensatz zur Leukämie ist das Hodenkarzinom, rechtzeitig diagnostiziert, fast zu 100\% heilbar. Die Verdachtsdiagnose erfordert nicht einmal eine Blutuntersuchung sondern kann in den meisten Fällen bereits durch das Abtasten des äußeren Genitale gestellt werden.

Mit Ihrer Aktion U25, der Name erinnert an die U-Untersuchungen bei Kindern und Jugendlichen, versucht die Deutsche Gesellschaft für Mann und Gesundheit auf diese Lücke aufmerksam zu machen. Das Untersuchungsprogramm umfasst neben einer körperlichen 
Untersuchung und einigen Laboruntersuchungen (Urin, Kleines Blutbild, Nüchternblutzucker, Blutfette und Testosteron) spezielle Fragebogen zur Familienanamnese, sportlicher Betätigung und Ernährungsgewohnheiten.

Die neuen S3-Leitlinien zur Früherkennung, Diagnose und Therapie des Prostatakarzinoms empfehlen eine Früherkennungsuntersuchung einschließlich einer PSA-Bestimmung ab dem vierzigsten Lebensjahr. Liegt der PSA-Wert unter $2 \mathrm{ng} / \mathrm{ml}$ reicht eine Kontrolluntersuchung alle 2 Jahre, liegt er höher als $2 \mathrm{ng} / \mathrm{ml}$ sollte eine jährliche Kontrolle erfolgen. Bei einem kontrollierten PSA-Wert von $\geq 4 \mathrm{ng} / \mathrm{ml}$ bei der erstmaligen Früherkennungsuntersuchung, einem suspekten digital-rektalen Tastbefund oder einem auffälligen PSA-Anstieg (ohne Wechsel des Bestimmungsverfahrens und unter Berücksichtigung der intraindividuellen Variabilität) ist eine ultraschallgesteuerte 10bis 12-fach Biopsie der Prostata indiziert. Der Prostatakrebs ist zwar der häufigste Krebs des Mannes. Bei einer rechtzeitigen Diagnose ist jedoch eine Heilung möglich.

Bezüglich der Häufigkeit urologischer Tumorerkrankungen kommt an zweiter Stelle beim Mann das Blasenkarzinom mit 18.850 und an dritter Stelle das Nierenzellkarzinom mit 10.300 Neuerkrankungen im Jahr. Auch hierfür werden in den urologischen Praxen wenig aufwendige Früherkennungsuntersuchungen angeboten. Nierentumore lassen sich bereits bevor sie symptomatisch werden (Makrohämaturie) im Ultraschall erkennen, für die Früherkennung des Urothelkarzinoms gibt es spezielle Urintests (NMP22, BTA-Test). Letzteres ist vor allem für sogenannte Risikogruppen (Raucher, Chemiearbeiter) besonders wichtig.

\subsection{Altersbedingte biologische Veränderungen}

Altersbedingte biologische Veränderungen beeinflussen natürlich auch die männliche Lebensqualität. Seit einigen Jahren wird kontrovers diskutiert, welche Bedeutung hierbei hormonelle Veränderungen haben. Während es bei der Frau durch die Einstellung der Eierstocksfunktion in der Menopause zu erheblichen hormonellen Umstellungen kommt, vollziehen sich die Veränderungen beim Mann ab dem vierzigsten Lebensjahr nur allmählich und unterliegen einer starken Varianz.

Statistisch sinkt das biologisch verfügbare freie Testosteron jährlich um 1,2\% ab, während gleichzeitig ein Anstieg des Sexualhormonbindenden Clobulins (SHBC) zu verzeichnen ist (Vermeulen u Kaufmann, 1995). Ferner ist bei älteren Männern ein Rückgang des morgendlichen Testosteronanstiegs zu beobachten, welcher auf einen Rückgang der LH-Pulsfrequenz zurückgeführt wird (Bremner et al. 1983). Als weitere Ursache wird eine Abnahme der Leydigzellen angenommen. In diesem Zusammenhang wurde der Begriff Late Onset Hypogonadism kreiert. Dieser Testosteronabsenkung werden eine ganze Reihe von organischen Veränderungen zugeschrieben wie die Zunahme des abdominellen Fetts, eine verringerte Muskelstärke, verminderter Bartwuchs, Osteoporose, Insulinresistenz und Arteriosklerose.

Einige Untersuchungen scheinen einen Zusammenhang mit der sog. PADAM-Symptomatik (PADAM = Partielles Androgendefizit des Alternden Mannes) wie nachlassende Libido, Erektionsstörungen, Müdigkeit, Abgeschlagenheit, Stimmungsschwankungen nahezulegen (Zitzmann et al. 2006). Andererseits sind diese Beschwerden auch bei vielen Männern mit völlig normalen, ja sogar mit besonders ho- 
hen Testosteronspiegeln $\mathrm{zu}$ finden (Thiele et al. 2001). Es gibt allerdings Hinweise, dass sowohl das metabolische Syndrom als auch die Entwicklung eines latenten Diabetes mit einem niedrigen Testeronspiegel korrelieren (Selvin et al. 2007). Ob unter diesem Aspekt eine Testosteronsubstitution sinnvoll ist, müssen weitere Untersuchungen zeigen. Entscheidet man sich im konkreten Fall für eine hormonergänzende Behandlung, sollten supraphysiologische Spiegel vermieden und regelmäßige Kontrolluntersuchungen der Prostata sowie Laborkontrollen (Testosteron, PSA, KBB, Leberwerte) durchgeführt werden.

\section{Literatur}

Arbeitsgemeinschaft Bevölkerungsbezogener Krebsregister in Deutschland (Hrsg.) (2004) Krebs in Deutschland, 4. überarbeitete, aktualisierte Ausgabe, Saarbrücken

Beier KM, Bosinski HAG, Loewit K (2005) Sexualmedizin. München, Jena: Urban \& Fischer; 2. Auflage

Berberich HJ, Ludwig M (2004) Psychosomatische Aspekte des chronischen Beckenschmerzes, Urologe A 43, 254-260

Brähler E, Stöbel-Richter Y (2002) Familienfeindlicher Zeitgeist? Reproduktionsmedizin 18, 276-282

Braun M, Wassmer G, Klotz T, Reifenrath B, Mathers M, Engelmann U (2000) Epidemiology of erectile dysfunction: results of the ,Cologne Male Survey'. Int I Impot Res 12, 305-311

Bremner W), Viteollo MV, Prinz PN (1983) Loss of circadian rhythmicity in blood testosterone levels with aging in normal men. I Clin Endocrinol Metab 56, 1278-1281

Feldmann HA, Goldstein I, Hatzichristou DG, Krane RI, Mc Kinlay JB (1994) Impotence and its medical and psychological correlates: results of the Massachusetts Male Aging Study. J Urol 151, 54-61

Gingell C, CrosbyD, Caroll R (2001) Review of the complications an medicolegal implications of vasectomy, Postgrad Med I 77, 656-659

Laumann EO, Paik A, Rosen RC (1999) Sexual dysfunction in the United States: prevalence and predictor. JAMA 281 537-544

Muller IE, Mittleman MA, Maclure M (1996) Triggering myocardial infarction by sexual activity. JAMA 275, 14051409

Selvin E, Feinleib M, Zhang L, Rohrmann S, Rifai N, Nelson WG, Dobs A, Basaria S, Golden SH, Platz EA (2007) Androgens and diabetes in men: results from the Third National Health and Nutrition Examination Survey (NHANES III) Diabetes Care 30, 1683

Speed TG, van Langen H, Meuleman E (2003) The risk of coronary heart desease in men with erectile dysfunction, Eur Urol 44, 366-370

Thiele A, Degenhardt A, Jaursch-Hancke C (2001) Bewältigung körperlicher Altersveränderungen bei gesunden Männern. In: Brähler E, Kupfer I (Hrsg.) Mann und Medizin, Göttingen, Hogrefe

Vermeulen A, Kaufmann IM (1995) Aging of Hypothlamo-pituitary-testicular axis in men, Hormone Research 43, $23-28$

Waldinger MD (2002) The neurobiological approach to premature ejaculation. J Urol 168, 2359-2367

Zitzmann M, Faber S, Nieschlag E (2006) Association of Specific Symptoms and Metabolic Risks with Serum Testosterone in Older Men. I Clin Endocrinol Metab 91, 4335-4343 